\title{
Prevalence of Cancers of Female Organs among Patients with Diabetes Type 2 in Kelantan, Malaysia: Observations over an 11 Year Period and Strategies to Reduce the Incidence
}

\author{
Nur Asyilla Che Jalil, Anani Aila Mat Zin, Nor Hayati Othman*
}

\begin{abstract}
Introduction: Kelantan is one of the states in Malaysia which has a high prevalence of type 2 diabetes (DM2). Other than with endometrial carcinoma, the association of DM2 with particular female cancers is not known. Objective: To determine the proportion of breast, cervical, ovarian and endometrial cancers among females with DM2 diagnosed in Hospital Universiti Sains Malaysia (HUSM) over an 11 year period. Materials and Methods: All histologically confirmed cases of breast, endometrial, cervical and ovarian carcinomas admitted to the Hospital were included in the study. The patient diabetic status was traced from the hospital medical records. Results: There was a total of 860 cases of breast, cervical, ovarian and endometrial carcinomas over this period. Breast carcinoma was the commonest, accounting for $437 / 860(50.8 \%)$ followed by cervix, $159 / 860$ (18.5\%), ovarian, 143/860 (16.6\%) and endometrial carcinomas, 121/860 (14.1\%). Out of these, 228/860 (26.5\%) were confirmed diabetics. Endometrial carcinoma patients showed the highest proportion being diabetics, $42.1 \%$ (51/121), followed by ovarian cancer, $25.9 \%$ (37/143), breast carcinoma, $23.6 \%(103 / 437)$ and cervical cancer 23.3\% (37/159). Conclusions: There is a significant proportion of DM2 among women with these four cancers, endometrial carcinoma being the highest followed by ovarian, breast and cervical carcinoma. The rising trend of these four cancers is in tandem with an increasing trend of DM2 in the community. In populations where diabetes is prevalent, screening for epithelial cancers should be rigourous. Diabetic clinics should include screening for these cancers among their female patients and gynecology clinics should screen the women they treat for their diabetes status.
\end{abstract}

Keywords: Female cancers - diabetes type 2 - Kelantan - increasing trend

Asian Pac J Cancer Prev, 16 (16), 7267-7270

\section{Introduction}

Kelantan, a state in the north eastern region of Malaysia records the highest prevalence of diabetes in the country with overall prevalence at $10.5 \%$ and pre-diabetic state at $16.5 \%$ (Zaini, 2000). This state also has high prevalence of obesity and the women has higher impaired glucose tolerance then men (Mafauzy et al., 1999). Obesity and diabetes have parallel relationship with cancer and the cancer burden in the state is seen to be escalating (Othman et al., 2008). Colorectal cancers among the population have been shown to have strong relationship with Diabetes Mellitus Type 2 (DM2) (Othman and Zin, 2008). However no studies on the association of DM2 and female cancers reported from a community which is prevalent with the disease.

There are many accumulating evidences showing that hyperinsulinemia in DM2 promotes carcinogenesis. In a large prospective study in Japan, patients with DM2 are at increased risk of total cancers (Inoue et al., 2006a). Some cancers develop more commonly in patients with
DM2 such as liver, pancreas, endometrial, colon, breast, bladder and lung (Vigneri et al., 2009). Ovarian cancer risk is significantly high in patients who are diagnosed diabetes at age less than 30 years (Swerdlow et al., 2005).

Epidemiological study shows that mortality rate for cancer patients with pre-existing diabetes is moderately increased compared to non diabetic patients (Vigneri et al., 2009). Hyperinsulinemia in diabetic patients promotes mammary carcinogenesis by increased IGF-1 level (Kulie et al., 2011). Women with Pre-existing DM2 has a $37 \%$ increase of all-cause mortality risk for women with breast cancer (Zhou et al., 2015).

The literature on association of cancer with diabetes mainly focus on epthelial cancers such as breast, endometrium, pancreas and hepatocellular carcinoma. The relationship between diabetes and risk of cervical cancer has not been extensively studied. Cervical cancer mortality is higher in obese women and are strongly associated with diabetes (Calle et al., 2003). Chronic diseases such as DM2 have become a barrier for women to undergo screening procedure of Pap smear and mammography. This factor is 
Nur Asyilla Che Jalil et al

believed to contribute to low compliance to early screening to prevent invasive cancer (Kiefe et al., 1998). In a study from Kelantan only $5.0 \%$ of cervical cancer patients have had pap smears within 3 years proceeding to cancer development (Othman et al., 2009).

Though the association of diabetes and cancer is strong some of these studies lack robustness (Tsilidis et al., 2015). One large ppopulation-based cohort study in Sweden showed lack of an association between DM2 and ovarian cancer (Weiderpass et al., 2002). Other studies also did not support the hypothesis that diabetes is a risk factor for epithelial ovarian cancer (Adler et al., 1996). However, diabetes in ovarian cancer is strongly associated with poor prognosis (Bakhru et al., 2011). In a large scale population based cohort study in Japan, there is a borderline significant in incidence of ovarian cancer and DM2 (Inoue et al., 2006a). In view that Kelantan has high prevalence of obesity and diabetes, we wanted to investigate if breast, endometrial, cervical and ovarian carcinoma have association with DM2.

\section{Materials and Methods}

All data on histologically diagnosed cancer cases of breast, endometrial, cervical and ovarian carcinoma in Hospital Universiti Sains Malaysia (HUSM) from 2000 to 2011 were retrieved from the computerized system in pathology department. Patients' diabetic status was obtained from the hospital medical records. Data was scrutinized to avoid duplicate entries. The data were analysed using SPSS 20.0

\section{Results}

A total of 860 cases of breast, cervical, ovary and endometrial carcinoma were included. In descending order; breast carcinoma 437/860 (50.8\%), cervical carcinoma 159/860 (18.5\%), ovarian carcinoma 143/860) (16.6\%) and endometrial carcinoma 121/860 (14.1\%). The age range of the patients 288 were from 21 to 93 years. The diabetic status of these patients were known in 791/860 (92.0\%) cases of which 228 was positive and 563 cases were negative. In 69 patients, the diabetes status was not known.

The trend of these four cancers was rising over the years with breast showing a steeper rise (Figure 1). Endometrial carcinoma patients showed the highest for being diabetic, 51/121 (42.1\%) followed by ovarian carcinoma $37 / 143(25.9 \%)$, breast carcinoma 103/437 (23.6\%) and cervical carcinoma 37/159 (23.3\%) (Figure 2). Of the 288 patients who were known positive for DM2, $45 \%(103 / 288)$ of the patients had breast carcinoma, $23 \%$ $(52 / 288)$ had endometrial carcinoma and both ovarian and cervical carcinoma were 16\% (37/288). The proportion of these four female cancers known positive for DM2 was also showing rising trend compared to those negative for DM2 (Figure 3).

The peak age of the patients included in this study was $53.37 \pm 11.96($ mean $\pm S D)$. The youngest age at presentation was 21 years old (ovarian carcinoma) while the oldest age at presentation was 93 years old (cervical carcinoma).

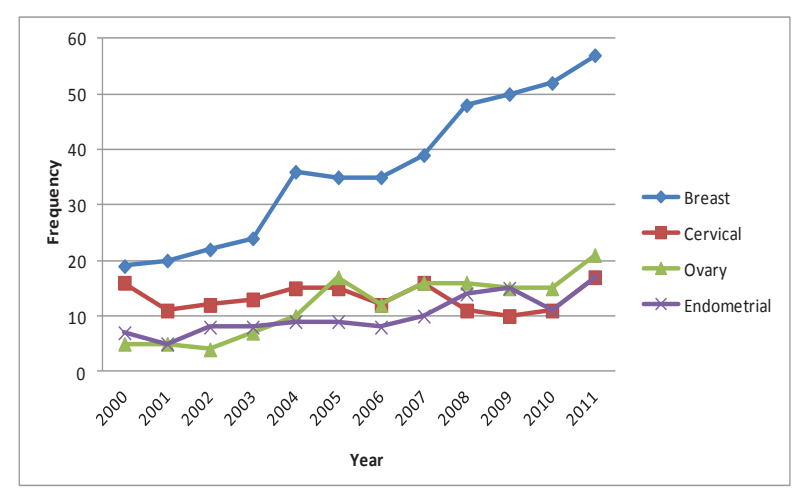

Figure 1. Pattern of Breasts, Cervical, Ovary and Endometrial Carcinoma Diagnosed in HUSM over the 11-year Period; 2000-2011

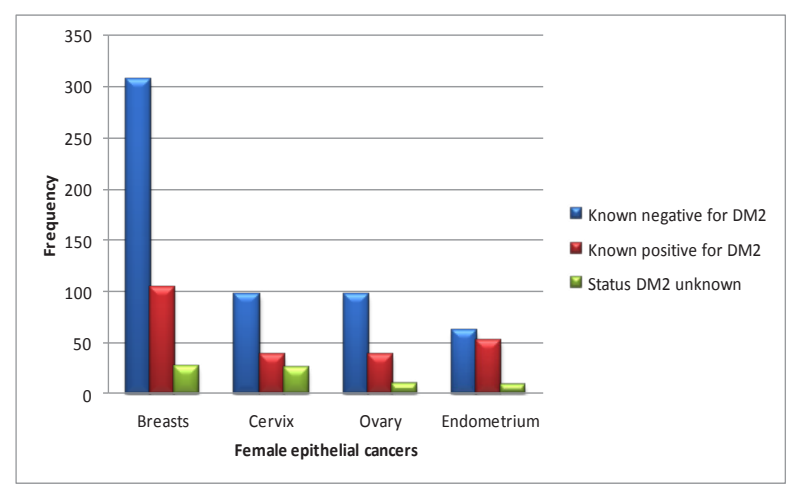

Figure 2. The Frequency of Breast, Cervical, Ovarian and Endometrial Carcinoma with Known Positive for Diabetes Mellitus Type 2 (DM2), known Negative for DM2 and Unknown Status in HUSM, 2000-2011

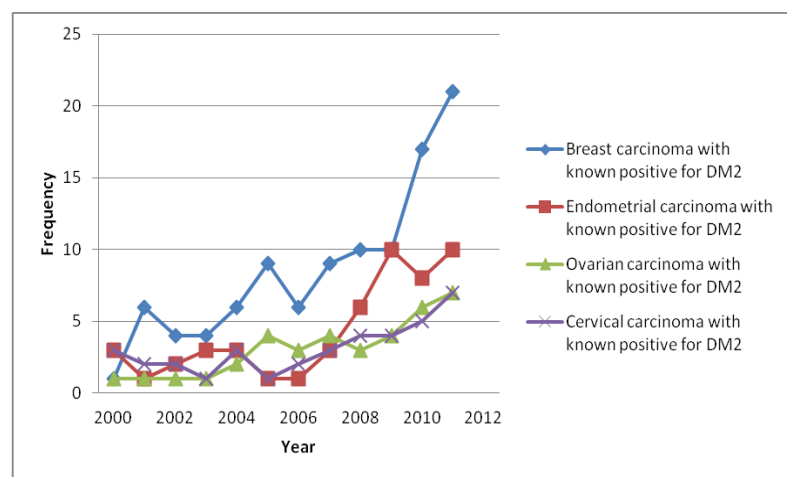

Figure 3. Pattern of Breasts, Cervical, Ovary and Endometrial Carcinomas with known Positive for Diabetes Mellitus Type 2 (DM2) in HUSM, 2000-2011

The young patient had a family history of diabetes while the old lady did not. According to ethnic distribution, the majority of cases were Malays, which made up of $84.8 \%$ (730/860) of study population and the proportion of Malay patients who were diabetic was $28 \%(205 / 730)$ while the Chinese though contributed $12.8 \%(110 / 860)$ of the total number of patients, the proportion of them being diabetic was $17 \%$ (19/110). Indians patients being diabetics was higher than the Chinese at $25 \%(1 / 4)$ and they make up only $0.5 \%$ (4/860) of total patients. Other ethnic minority such as the Thais comprised of $1.9 \%(16 / 860)$ of the total number and the proportion of them being diabetic was also high at $19 \%(3 / 16)$. 
Majority, $56.7 \%$ (248/437) of the breast cancer (stage IV) and ovarian carcinoma $37.0 \%(53 / 143)$ (stage 111) presented at advanced stage. While the majority $35.2 \%$ (56/159) of cervical carcinoma patients presented at stage II. Endometrial carcinoma patients presented at early stage (stage I) $30.6 \%(37 / 121)$ in the majority.

\section{Discussion}

Gynaecological malignancy including breast cancers accounts for $15-20 \%$ of all cancers in women. In this study which covers an 11-year period, a total of 860 cases of histologically proven breasts, cervical, ovary and endometrial carcinoma were encountered. This figure excludes cases referred to HUSM from other hospitals. At the same time period HUSM records a total of 5114 cancer cases making the proportion of women cancers in our community $16.8 \%$ comparable to the proportion seen in many countries. Among these carcinomas, the highest proportion was breast carcinoma followed by cervical, ovarian and endometrial carcinoma. The trend of these four female cancers in HUSM showed a slow rise akin to the rise of other cancers seen in this hospital (Othman et al., 2008). It is more worrisome to note that patients as young as 21 years old been diagnosed to have cancer. In order to combat the rising cancer trents, two strategies are needed; reduce the prevalence of diabetes and institute rigorous cancer screening programs among women. Both issues are yet to be optimally addressed in Malaysia.

In Malaysia, several implementation stategies have been put forward to increase breasts (Dahlui et al., 2011) and cervical (Othman and Mohamad Zaki, 2014) cancer detection. However, not all women voluntarily undergo the screening procedure such as mammography or pap smear. The barriers to early detection are multiple including geographical isolation, lack of education (Othman and Rebolj, 2009, Breen and Kessler, 1994) and lack of autonomous decision-making in the male-dominated society (Yip and Taib, 2014). Furthermore, they tend to believe that traditional healer is better (Yip and Taib, 2014). The long waiting time in goverment hospitals also might influence the decision not to come to hospital early (Othman and Rebolj, 2009).

Ovarian and endometrial carcinoma do not have recognized screening program in Malaysia and in many countries worldwide. Though the risk of endometrial carcinoma among diabetics women is well known (Tsilidis et al., 2015) (Salazar-Martínez et al., 2000), its association with ovarian cancer (Inoue et al., 2006b; Shah et al., 2014 ) is less so. In the majority, these deseases remain sub-clinical until advanced stage. The lack of specific screening probably could be replaced with regular blood glucose level to detect early detection of DM2 and if they are already diabetics, to ensure the disease is under control. With lifestyle modification and pharmacotherapy, DM2 is a preventable and treatable disease. The risk of cancer development may also be lowered by these strategies. Cancer screening should be one of the steps to be instituted in diabetic clinics and diabetic screening in gynaecology clinics.

The association of obesity, diabetes and hypertension with the risk of endometrial cancer is well known. These factors possibly exert their effects by indirect influences on oestrogen levels. The overall risk of endometrial carcinoma with unopposed estrogenic stimuli is six-fold higher compared to those without (Antunes et al., 1979).

Carcinogenesis is a complex process. Normal cells need to undergo multiple genetic hits in order for neoplastic change to occur involving 'initiation', 'promotion' and 'progression' steps (Kumar et al., 2012). Diabetes may influence the cancer formation by contributing in any of above steps. Possible mechanisms for a direct link between diabetes and cancer include hyperinsulinemia, hyperglycemia, and inflammation (Pollak, 2008). Apart from the direct effects of insulin on cancer cells, it is possible that hyperinsulinemia could promote carcinogenesis indirectly through its effects on IGF. Multiple signaling pathways are activated after insulin receptors or IGF-I receptors interact with their ligands. High IGF-1 level can inhibit apoptosis of mutated cells and enhance proliferation of the mutated DNA which lead to cancer formation (Pollak, 2008).

It is estimated that there would be 2.48 million diabetic patients in Malaysia by 2030 and Kelantan state is one with the highest prevalence in the country (Mafauzy, 2006). Women of this state have been noted to have a higher risk to develop metabolic diseases (Mohamed et al., 2013). With such a high prevalence of diabetes and those at risk of getting the disease, the increased prevalence of female cancers in Kelantan state is not a surprise. Our study shows that endometrial carcinoma patients showed the highest for being diabetic followed by ovarian, breast and cervical carcinomas. To the best of our knowledge this is first study on the association DM2 and female cancers in Kelantan. Our data shows a much higher percentage on breast cancer cases with DM2 (23.6\%) compared to the study by Michels et al, where only $3.6 \%$ the breast cancer in their series noted to be diabetic in a 10 years follow up period (Michels et al., 2003). Women are at 2-3 fold risk of getting endometrial cancer when they are diabetics compared to the general population (Lindemann et al., 2008) and more than 6 fold when they are also obese (Friberg et al., 2007).

The association of ovarian carcinoma with diabetes is controversial from lack of association in one study in Sweden (Weiderpass et al., 2002) to a borderline significant association in another large study in Japan (Inoue et al., 2006b). Patients who are diabetics have worse prognosis of this cancer compared to those without (Bakhru et al., 2011).

Unlike the three cancers mentioned, cervical carcinoma is due to infection by HPV virus where the oncogenic protien, E7 and E6 can alter the cell cycle to inhibit apoptosis (Kumar et al., 2012). However, the evidence does not implicate HPV as the only factor. A high percentage of young women who are infected with one or more HPV types during their reproductive years do not develop cancer. Other factors such as the immune status, nutrition, DM2 and obesity could be the contributing factors. It has been shown that cervical cancer is higher in obese and diabetic women (Calle et al., 2003). Chronic diseases in women often become the barrier to screening 
Nur Asyilla Che Jalil et al

for cervical cancer (Kiefe et al., 1998) thus a slight higher incidence among them.

There were two patients in our series who had cancer outside the usual age range for the particular cancer. One was a 21 years old who had ovarian carcinoma and the other was a 93 years old who had cervical carcinoma. The young patient had a family history of DM2 while the old lady did not and was non-diabetic.

In conclusion there is a rising trend of female cancers seen over the eleven year period in Kelantan. Many of these patients are diabetics. In descending order; endometrial carcinoma patients showed the highest for being diabetic, followed by ovarian, breast and cervical carcinomas. This rising trend of female cancers is also accompanied by increasing trend of DM2 in the community. In a community where diabetes is prevalent, screening for epithelial cancers should be rigourous. Diabetic clinics should include screening for these cancers in their diabetic patients follow-up protocol and gynaecology clinics should screen for diabetes status of all women they see.

\section{References}

Adler AI, Weiss NS, Kamb ML, et al (1996). Is diabetes mellitus a risk factor for ovarian cancer? A case-control study in Utah and Washington (United States). Cancer Causes Control, 7, 475-8.

Antunes CM, Stolley PD, Rosenshein NB, et al (1979). Endometrial cancer and estrogen use: Report of a large case-control study. New England J Medicine, 300, 9-13.

Bakhru A, Buckanovich RJ, Griggs JJ (2011). The impact of diabetes on survival in women with ovarian cancer. Gynecol Oncol, 121, 106-11.

Breen N \& Kessler L (1994). Changes in the use of screening mammography: evidence from the 1987 and 1990 National Health Interview Surveys. Am J Public Health, 84, 62-67.

Calle E E, Rodriguez C, Walker-Thurmond K, et al (2003). Overweight, obesity, and mortality from cancer in a prospectively studied cohort of U.S. adults. N Engl J Med, 348, 1625-38.

Dahlui M, Ramli S, Bulgiba AM (2011). Breast cancer prevention and control programs in Malaysia. Asian Pac J Cancer Prev, 12, 1631-4.

Friberg E, Mantzoros CS, Wolk A (2007). Diabetes and risk of endometrial cancer: a population-based prospective cohort study. Cancer Epidemiol Biomarkers Prev, 16, 276-80.

Inoue M, Iwasaki M, Otani T, et al (2006a). Diabetes mellitus and the risk of cancer: results from a large-scale populationbased cohort study in Japan. Arch Intern Med, 166, 1871-7.

Inoue M, Iwasaki M, Otani T, et al (2006b). Diabetes mellitus and the risk of cancer: results from a large-scale populationbased cohort study in Japan. Arch Intern Med, 166, 1871.

Jan Mohamed HJ, Mitra AK, Zainuddin LR, et al (2013). Women are at a higher risk of metabolic syndrome in rural Malaysia. Women Health, 53, 335-48.

Kiefe CI, Funkhouser E, Fouad MN, et al (1998). Chronic disease as a barrier to breast and cervical cancer screening. J Gen Intern Med, 13, 357-65.

Kulie T, Slattengren A, Redmer J, et al (2011). Obesity and women's health: an evidence-based review. J Am Board Fam Med, 24, 75-85.

Kumar V, Abbas AK, Aster JC (2012) Robbins basic pathology, Elsevier Health Sciences.

Lindemann K, Vatten LJ, Ellstrom-Engh M, et al (2008). Body mass, diabetes and smoking, and endometrial cancer risk: a follow-up study. Br J Cancer, 98, 1582-5.

Mafauzy M (2006). Diabetes mellitus in Malaysia. Med $J$ Malaysia, 61, 397-8.

Mafauzy M, Mokhtar N, Mohamad WB, et al (1999). Diabetes mellitus and associated cardiovascular risk factors in northeast Malaysia. Asia Pac J Public Health, 11, 16-9.

Michels KB, Solomon CG, Hu FB, et al (2003). Type 2 diabetes and subsequent incidence of breast cancer in the Nurses' Health Study. Diabetes Care, 26, 1752-8.

Othman NH, Devi BC, Halimah Y (2009). Cervical cancer screening: patients understanding in major hospitals in Malaysia. Asian Pac J Cancer Prev, 10, 569-74.

Othman NH, Mohamad Zaki FH (2014). Self-collection tools for routine cervical cancer screening: a review. Asian Pac J Cancer Prev, 15, 8563-9.

Othman NH, Nor ZM, Biswal BM (2008). Is Kelantan joining the global cancer epidemic?--experience from hospital Universiti Sains Malaysia; 1987-2007. Asian Pac J Cancer Prev, 9, 473-8.

Othman NH, Rebolj M (2009). Challenges to cervical screening in a developing country: The case of Malaysia. Asian Pac J Cancer Prev, 10, 747-52.

Othman N H \& Zin AA (2008). Association of colorectal carcinoma with metabolic diseases; experience with 138 cases from Kelantan, Malaysia. Asian Pac J Cancer Prev, 9,747-51.

Pollak M (2008). Insulin and insulin-like growth factor signalling in neoplasia. Nat Rev Cancer, 8, 915-28.

Salazar-Martínez E, Lazcano-Ponce EC, Lira-Lira GG, et al (2000). Case-control study of diabetes, obesity, physical activity and risk of endometrial cancer among Mexican women. Cancer Causes \& Control, 11, 707-711.

Shah MM, Erickson BK, Matin T, et al (2014). Diabetes mellitus and ovarian cancer: more complex than just increasing risk. Gynecol Oncol, 135, 273-7.

Swerdlow AJ, Laing SP, Qiao Z, et al (2005). Cancer incidence and mortality in patients with insulin-treated diabetes: a UK cohort study. Br J Cancer, 92, 2070-5.

Tsilidis KK, Kasimis JC, Lopez DS, et al (2015). Type 2 diabetes and cancer: umbrella review of meta-analyses of observational studies. BMJ, 350, 7607.

Vigneri P, Frasca F, Sciacca L, et al (2009). Diabetes and cancer. Endocr Relat Cancer, 16, 1103-23.

Weiderpass E, Ye W, Vainio H, et al (2002). Diabetes mellitus and ovarian cancer (Sweden). Cancer Causes Control, 13, 759-64.

Yip CH, Taib NA (2014). Breast health in developing countries. Climacteric, 17, 54-9.

Zaini A (2000). Where is Malaysia in the midst of the Asian epidemic of diabetes mellitus? Diabetes Res Clin Pract, 50, 23-8.

Zhou Y, Zhang X, Gu C, et al (2015). Diabetes mellitus is associated with breast cancer: systematic review, metaanalysis, and in silico reproduction. Panminerva Med, 57, 101-8. 